\title{
OPTIMAL EXPERIMENT DESIGN IN CLOSED LOOP
}

\author{
Henrik Jansson * Håkan Hjalmarsson * \\ * KTH, Signals, Sensors and Systems, S-100 44 Stockholm, \\ Sweden. henrik.janssonas3.kth.se
}

\begin{abstract}
In this contribution we extend a recently developed framework for open loop input design to closed loop experiment design. More specifically, for the very common situation of a fixed controller during the identification experiment, the framework is extended to the design of an optimal reference signal spectrum and also to cope with closed loop signal constraints. In a further extension also the regulator is included in the experiment design leading to a very general experiment design framework.

Copyright (c)IFAC 2005
\end{abstract}

Keywords: identification, experiment design, convex optimization

\section{INTRODUCTION}

In system identification, there is a strong relation between the achieved accuracy of obtained models and the experiment conditions such as external excitation signals. This fact received considerable interest in the 1970's, where (Goodwin and Payne, 1977) and (Zarrop, 1979) are good introductions to optimal input design in open loop. Typical design problems in this context correspond to non-convex programs and, hence, computational aspects have limited the applicability of optimal input design. One way of avoiding this has been to rely on high-order expressions for the model accuracy (Ljung, 1985; Yuan and Ljung, 1985; Gevers and Ljung, 1986; Hjalmarsson et al., 1996; Forssell and Ljung, 2000), which beside computational favors provides a certain robustness against the properties of the underlying system. Despite that these high-order results can be quite accurate even for model orders as low as two, see (Ljung, 1985; Ljung, 1999), it is easy to construct examples where they fail, for arbitrarily high orders see (Ninness and Hjalmarsson, 2002). Furthermore, these results are not suitable for handling frequency wise constraints, which e.g. is an important ingredient in many robust control applications.

Motivated by these limitations, there has been renewed interest in input design for models of finite order leading to the contributions (Lindqvist and Hjalmarsson, 2000; Lindqvist and Hjalmarsson, 2001; Hildebrand and Gevers, 2003), (Hildebrand and Gevers, 2003), (Bombois et al., 2004b) and (Jansson and
Hjalmarsson, 2004a), in which a new approach to solve input design problems has been opened up. It has been shown that a wide range of open loop input design problems are equivalent to convex programs. Today, there exist several powerful numerical methods to solve convex programs.

In the context of control applications, various arguments for the advantages of identification in closed loop has been brought forward. In (Gevers and Ljung, 1986) it was shown that for situations when the high order variance expression is valid, closed loop experiments under minimum variance control are optimal if the model is to be used for minimum variance control. For similar problem formulations it was shown in (Hjalmarsson et al., 1996) that it is possible to outperform any fixed input design by a sequence of closed loop designs, provided the experimentation time is long enough. Again relying on the high-order variance expression, (Forssell and Ljung, 2000) showed that, typically, closed loop experiments are optimal when the output variance is constrained during the experiments. In another line of research focusing on the bias error it has also been shown that closed loop experiments can be beneficial, see e.g. (Zang et al., 1995; Lee et al., 1993). Closed loop experimentation can also be motivated by practical arguments. Most industrial processes are being operated in closed loop and it is often not possible to open the loop. Therefore, identification experiments often have to be performed in closed loop with an existing controller in the loop. 
Considering the above, it is of interest to re-examine optimal closed loop experiment design for finite model order. In this contribution, we will continue the work in (Jansson and Hjalmarsson, 2004b; Jansson and Hjalmarsson, 2004a) and generalize these results to include feedback in the input. Some early contributions on experiment design in closed loop are $(\mathrm{Ng}$ et al., 1977b; Ng et al., 1977a; Gustavsson et al., 1977).

A brief review of parameter estimation is given in Section 2. Some relevant issues involved in typical design formulations are illustrated in Section 3. Experiment design with a fix controller is treated in Section 4. The case with the feedback mechanism included in the experiment design is treated in Sections 5-7. The paper is summarized in Section 8.

\section{PARAMETER ESTIMATION}

We will consider identification of time-discrete linear time-invariant systems within the prediction error framework, (Ljung, 1999). The true single input/single output system is modelled by

$$
y(t)=G(q, \theta) u(t)+H(q, \theta) e(t)
$$

where $G$ and $H$ are parametrized by the real valued parameter vector $\theta$. Furthermore, $y$ is the output and $u$ is the input and $e$ is zero mean white noise with variance $\lambda$. It is assumed that $G$ and $H$ have the rational forms

$$
G(q, \theta)=\frac{q^{-n_{k}} B(q, \theta)}{A(q, \theta)}, \quad H(q, \theta)=\frac{C(q, \theta)}{D(q, \theta)}
$$

where

$$
\begin{aligned}
& A(q, \theta)=1+a_{1} q^{-1}+\cdots+a_{n_{a}} q^{-n_{a}} \\
& B(q, \theta)=b_{0}+b_{1} q^{-1}+\cdots+b_{n_{b}} q^{-n_{b}} \\
& C(q, \theta)=1+c_{1} q^{-1}+\cdots+c_{n_{c}} q^{-n_{c}} \\
& D(q, \theta)=1+d_{1} q^{-1}+\cdots+d_{n_{d}} q^{-n_{d}}
\end{aligned}
$$

with $q$ being the delay operator. We will assume that there exists a description of the true system within the model class defined by $\theta=\theta_{o}$ and $\lambda=\lambda_{o}$. The onestep-ahead predictor for the model (1) is

$$
\begin{aligned}
\hat{y}(t, \theta) & =H^{-1}(q, \theta) G(q, \theta) u(t) \\
& +\left(1-H^{-1}(q, \theta)\right) y(t)
\end{aligned}
$$

and the prediction error is $\varepsilon(t, \theta)=y(t)-\hat{y}(t, \theta)$. The parameters are estimated with the prediction error method using a least mean square criterion to minimize the prediction error. The parameter estimate is

$$
\widehat{\theta}_{N}=\underset{\theta}{\arg \min } \frac{1}{2 N} \sum_{t=1}^{N} \varepsilon^{2}(t, \theta)
$$

where $N$ denotes the number of the data that is used for the estimation. Under mild assumptions the parameter estimate has an asymptotic distribution (Ljung, 1999) that obeys

$$
\begin{aligned}
& \sqrt{N}\left(\widehat{\theta}_{N}-\theta_{o}\right) \in \operatorname{AsN}(0, P) \\
& P=\lambda_{o}\left(\mathrm{E}\left\{\psi\left(t, \theta_{o}\right) \psi^{T}\left(t, \theta_{o}\right)\right\}\right)^{-1} \\
& \psi\left(t, \theta_{o}\right)=-\left.\frac{\partial}{\partial \theta} \varepsilon(t, \theta)\right|_{\theta=\theta_{o}}=\left.\frac{\partial}{\partial \theta} \widehat{y}(t \mid \theta)\right|_{\theta=\theta_{o}}
\end{aligned}
$$

Introduce $\chi_{o}(t)=\left[u(t) e_{o}(t)\right]^{T}$ where $e_{o}(t)$ represents the true noise source. Using (7) and (9) we obtain

$$
\psi\left(t, \theta_{o}\right)=\mathcal{F}\left(q, \theta_{o}\right) \chi_{o}(t)
$$

where $\mathcal{F}\left(q, \theta_{o}\right)=\left[\mathcal{F}_{u}\left(q, \theta_{o}\right) \mathcal{F}_{e}\left(q, \theta_{o}\right)\right]$ with

$$
\begin{aligned}
\mathcal{F}_{u}(q, \theta) & =\frac{1}{H(q, \theta)} \frac{\partial G(q, \theta)}{\partial \theta} \\
\mathcal{F}_{e}(q, \theta) & =\frac{1}{H(q, \theta)} \frac{\partial H(q, \theta)}{\partial \theta}
\end{aligned}
$$

In this paper we will assume that the input is generated as

$$
u(t)=-K(q) y(t)+r(t)
$$

where $r(t)$ is an external reference that is uncorrelated with the noise $e(t)$. The controller $K(q)$ is assumed to be linear and causal. Furthermore, all signals are assumed to have a spectral representation.

Based on (9)-(13), it is possible to express the inverse of $P$ in (9) as follows

$$
\begin{gathered}
P^{-1}=\frac{1}{2 \pi \lambda_{o}} \int_{-\pi}^{\pi} \mathcal{F}\left(\theta_{o}\right) \Phi_{\chi_{o}}\left(\theta_{o}\right) \mathcal{F}^{*}\left(\theta_{o}\right) d \omega \\
\Phi_{\chi_{o}}=\left[\begin{array}{cc}
\Phi_{u} & \Phi_{u e} \\
\Phi_{u e}^{*} & \lambda_{o}
\end{array}\right]
\end{gathered}
$$

Here $\Phi_{u}$ is the spectrum of the input and $\Phi_{u e}$ is the cross spectrum between $u$ and $e_{O}$. The expression (14) is very useful for experiment design purposes in closed loop operation. It shows exactly the influence of the input spectrum and the cross spectrum on the asymptotic parameter covariance matrix. This connection between the asymptotic covariance and these spectra will be further exploited in the subsequent sections.

\section{ISSUES ON OPTIMAL EXPERIMENT DESIGN}

If a model has been identified from experimental data it is inevitable that the model will contain errors. To evaluate the quality of the estimated models and the possible performance degradations that the model errors may induce, it is important to quantify them. We will assume that we have a full-order model structure and hence only variance errors occurs. Therefore, for large data lengths, the model error can be characterized by some function of the parameter covariance $P$. Some classical quality measures are the scalar functions $\operatorname{det}(P)$ and $\operatorname{Tr} W P$ where $W$ is a weighting matrix. Recently, several frequency by frequency conditions on the error of the frequency function estimate have been used in different optimal input design formulations, see e.g. (Hildebrand and Gevers, 2003; Bombois et al., 2004a; Jansson and Hjalmarsson, 2004b). One example is the following weighted variance criterion

$$
\frac{d G^{*}\left(\mathrm{e}^{j \omega}, \theta_{o}\right)}{d \theta} P\left(\Phi_{\chi_{o}}\right) \frac{d G\left(\mathrm{e}^{j \omega}, \theta_{o}\right)}{d \theta} \leq b(\omega)
$$

where the left hand side is a first order approximation of the variance of the frequency function estimate $\widehat{G}\left(\mathrm{e}^{j \omega}, \widehat{\theta}_{N}\right)$. The upper bound $b(\omega)$ specifies the important frequency bands wrt the quality of the model. 
Experiment design problems are often formulated as optimization programs that involve non-convex constraints, e.g. (16). A framework for handling such problems has recently been presented in (Jansson and Hjalmarsson, 2004b) and (Jansson and Hjalmarsson, 2004a) for design of inputs when the considered system is in open loop operation. The key to obtain tractable optimization problems in open loop is to impose suitable parametrizations of the input spectrum. Here we will generalize the results of (Jansson and Hjalmarsson, 2004b) and (Jansson and Hjalmarsson, 2004a) and include possible feedback in the input. The main difference between design in open loop compared to design in closed loop is the parametrizations of signal constraints and the inverse covariance matrix. Therefore, we will focus on these issues in this paper. For example, once we have retrieved a linear and finite parametrization of $P^{-1}$ it is straightforward to handle quality constraints as (16) by applying some of the methods that have been suggested for input design in open loop, $c f$. (Jansson and Hjalmarsson, 2004b).

\section{EXPERIMENT DESIGN IN CLOSED LOOP WITH A FIX CONTROLLER}

We will start with the simplest case where the controller is fix but where the spectrum of the reference signal is at the designer's disposal. We begin by observing that $\Phi_{\chi_{o}}$ can be written as

$$
\Phi_{\chi_{o}}=\left[\begin{array}{cc}
\left(\left|S_{o}\right|^{2} \Phi_{r}+\left|K S_{o} H\left(\theta_{o}\right)\right|^{2} \lambda_{o}\right) & \Phi_{u e} \\
\Phi_{u e}^{*} & \lambda_{o}
\end{array}\right]
$$

where $S_{o}=1 /\left(1+G\left(\theta_{o}\right) K\right.$ is the sensitivity function. We see that $\Phi_{\chi_{o}}$ is affine in the reference spectrum $\Phi_{r}$ and consequently, as is evidenced by (14), the inverse covariance matrix $P^{-1}$ is affine in the same quantity. With the input spectrum substituted for the reference spectrum, this is exactly the basis for the design techniques for open loop systems, e.g. the ones presented in (Jansson and Hjalmarsson, 2004b). It is, hence, straightforward to modify existing open loop design techniques to handle design of the reference spectrum when the controller is fix. This means that we can handle (see (Jansson and Hjalmarsson, 2004b) for details)

- any energy constraint on the reference spectrum of the form

$$
\frac{1}{2 \pi} \int_{-\pi}^{\pi}\left|W_{r}\left(e^{j \omega}\right)\right|^{2} \Phi_{r}(\omega) d \omega
$$

where $W_{r}$ is a stable transfer function. This includes $e . g$. input energy as well as output energy constraints.

- point-wise constraints

$$
\alpha_{r}(\omega) \leq \Phi_{r}(\omega) \leq \beta_{r}(\omega) \quad \forall \omega
$$

- general linear, possibly frequency dependent, functions of the asymptotic covariance matrix P, e.g. (16). It is also possible to use certain types of quality constraints that are guaranteed to hold in a confidence region.

\section{EXPERIMENT DESIGN IN CLOSED LOOP WITH A FREE CONTROLLER}

We will now generalize the scenario to the case where, in addition to the reference spectrum, also the feedback mechanism $K(q)$ in (13) can be chosen. We thus have both $\Phi_{r}$ and $K$ at our disposal. However, it will turn out to be more natural to instead use the input spectrum $\Phi_{u}$ and the cross spectrum $\Phi_{u e}$ as design variables. Since there is a one-to-one between these two sets of variables this imposes no restrictions. This will be the set-up in the remaining part of the paper.

\section{SPECTRUM REPRESENTATIONS}

We will briefly discuss some useful options to parametrize a spectrum. Generally, the spectrum (15) may be written

$$
\Phi_{\chi_{o}}=\sum_{k=-\infty}^{\infty} \tilde{c}_{k} \mathcal{B}_{k}
$$

for some basis functions $\left\{\mathcal{B}_{k}\right\}_{k=-\infty}^{\infty}$ which span $\mathcal{L}_{2}$. The matrix-valued coefficients $\tilde{c}_{k} \in \mathbb{R}^{2 \times 2}$ must be such that

$$
\Phi_{\chi_{o}}(\omega) \geq 0, \quad \forall \omega
$$

For the most common choice of basis functions, $\mathcal{B}_{k}\left(e^{j \omega}\right)=e^{-j \omega k}$, the coefficients $\tilde{c}_{k}$ have the interpretation as auto-correlations. Since $P^{-1}$ is linear in $\Phi_{\chi_{o}}$, it is natural to parametrize the spectrum in terms of the coefficients $\tilde{c}_{k}$. However, it is impractical to use an infinite number of parameters so the parametrization has to be restricted. In (Jansson and Hjalmarsson, 2004a), two different approaches are presented for this. They are denoted as "finite dimensional spectrum parametrization" and "partial correlation parametrization", respectively. Here we will shortly review these two concepts. For a more thorough treatment we refer to (Jansson and Hjalmarsson, 2004a).

\subsection{Finite dimensional spectrum parametrization}

The finite dimensional spectrum parametrization is based on

$$
\Phi_{\chi_{o}}=\sum_{k=-(M-1)}^{M-1} \tilde{c}_{k} \mathcal{B}_{k}
$$

for some positive integer $M$. Here one has to impose the condition (20) to ensure that $\Phi_{\chi_{o}}$ indeed is a spectrum. The KYP-lemma may be useful for this ((Jansson and Hjalmarsson, 2004b).

\subsection{Partial correlation parametrization}

As an alternative to (21), one may use a partial expansion

$$
\sum_{k=-(M-1)}^{M-1} \tilde{c}_{k} \mathcal{B}_{k}
$$

which may not be a spectrum in itself, but constrained such that there exists additional coefficients $\tilde{c}_{k}, k=$ 
$M, M+1, \ldots$ such that the expansion (19) satisfies the non-negativity condition (20). This approach, which we denote as "partial correlation parametrization", thus enables one to work with infinite dimensional expansions. When using a partial correlation parametrization one must ensure that there exists an extension $\tilde{c}_{M}, \quad \tilde{c}_{M+1}, \quad \tilde{c}_{M+2}, \ldots$ of the sequence $\tilde{c}_{0}, \tilde{c}_{1}, \ldots, \tilde{c}_{M-1}$ so that the corresponding basis function expansion (19) defines a spectrum.

In the next section we will show that both the spectrum parametrization (21) and the correlation parametrization (22) can be used to parametrize the inverse covariance matrix $P^{-1}$ and some signal constraints.

\section{PARAMETRIZATIONS OF THE COVARIANCE MATRIX AND SIGNAL CONSTRAINTS}

\subsection{A parametrization in terms of partial correlations}

Since the elements of $\mathcal{F}$ span a linear subspace, it follows that the set of all covariance matrices can be parametrized in terms of finite dimensional parametrizations of $\Phi_{\chi_{o}}$. Here we will characterize one such parametrization. This parametrization is based on an idea originally presented in (Payne and Goodwin, 1974) with further developments in (Zarrop, 1979) and (Stoica and Söderström, 1982) for input design in open loop. Here we will present the generalization to experiment design in closed loop.

The objective is to obtain a linear and finite parametrization of $P^{-1}$ and the weighted output energy defined by

$$
\frac{1}{2 \pi} \int_{-\pi}^{\pi}\left|W_{y}\left(\mathrm{e}^{j \omega}\right)\right|^{2} \Phi_{y}(\omega) d \omega
$$

where $W_{y}\left(\mathrm{e}^{j \omega}\right)$ is a stable scalar transfer function. The starting point is the spectrum of the output defined by

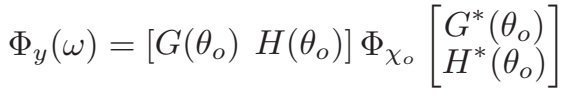

Now introduce the spectrum $\widetilde{\Phi}_{\chi_{o}}$ defined by

$$
\frac{\widetilde{\Phi}_{\chi_{o}}}{\left|W_{y}\right|^{2}}=\left[\begin{array}{cc}
G\left(\theta_{o}\right) & 0 \\
0 & H\left(\theta_{o}\right)
\end{array}\right] \Phi_{\chi_{o}}\left[\begin{array}{cc}
G^{*}\left(\theta_{o}\right) & 0 \\
0 & H^{*}\left(\theta_{o}\right)
\end{array}\right]
$$

Notice that

$$
\left|W_{y}\right|^{2} \Phi_{y}=\left[\begin{array}{ll}
1 & 1
\end{array}\right] \widetilde{\Phi}_{\chi_{o}}\left[\begin{array}{l}
1 \\
1
\end{array}\right]
$$

Now let $\widetilde{\mathcal{F}}=\left[\mathcal{F}_{u} / G, \mathcal{F}_{e} / H\right] / W_{y}$ and parametrize $\widetilde{\mathcal{F}}$ on the form

$$
\widetilde{\mathcal{F}}\left(\mathrm{e}^{j \omega}\right)=\frac{1}{\mathcal{F}_{D}\left(\mathrm{e}^{j \omega}\right)} \sum_{k=0}^{m} M_{\mathcal{F}}(k) \mathrm{e}^{-k j \omega}
$$

where the scalar transfer function $\mathcal{F}_{D}\left(\mathrm{e}^{j \omega}\right)$ corresponds to the least common denominator to $\widetilde{\mathcal{F}}\left(\mathrm{e}^{j \omega}\right)$ and where $M_{\mathcal{F}}(k), k=1, \ldots, m$ are some real matrices. Then introduce the parametrization

$$
\widetilde{\Phi}_{\chi_{o}}(\omega)=\left|\mathcal{F}_{D}\left(\mathrm{e}^{j \omega}\right)\right|^{2} \sum_{k=-\infty}^{\infty} R_{k} \mathrm{e}^{-k j \omega}
$$

i.e. a parametrization of the form (19) with $\mathcal{B}_{k}=$ $\left|\mathcal{F}_{D}\left(\mathrm{e}^{j \omega}\right)\right|^{2} \mathrm{e}^{-k j \omega}$. Now it is straightforward to rewrite $P^{-1}$ as a linear function in the auto-correlations $R_{k}$ as follows

$$
\begin{aligned}
& P^{-1}=\frac{1}{2 \pi \lambda_{o}} \int_{-\pi}^{\pi} \tilde{\mathcal{F}}\left(\theta_{o}\right) \widetilde{\Phi}_{\chi_{o}}\left(\theta_{o}\right) \widetilde{\mathcal{F}}^{*}\left(\theta_{o}\right) d \omega \\
& =\sum_{k=0}^{m} \sum_{l=0}^{m} \frac{1}{2 \pi \lambda_{o}} \int_{-\pi}^{\pi} \frac{M_{\mathcal{F}}(k) \Phi_{\chi_{o}} M_{\mathcal{F}}^{T}(l)}{\mathcal{F}_{D}\left(\mathrm{e}^{j \omega}\right) \mathcal{F}_{D}^{*}\left(\mathrm{e}^{j \omega}\right)} \mathrm{e}^{(l-k) j \omega} \\
& =\sum_{k=0}^{m} M_{\mathcal{F}}(k) R_{0} M_{\mathcal{F}}^{T}(k) \\
& \quad+\sum_{k=1}^{m} \sum_{l=0}^{m-k} M_{\mathcal{F}}(l) R_{k} M_{\mathcal{F}}^{T}(l+k) \\
& \quad+\sum_{k=1}^{m} \sum_{l=0}^{m-k} M_{\mathcal{F}}(l+k) R_{k}^{T} M_{\mathcal{F}}^{T}(l)
\end{aligned}
$$

Remark: Notice that all covariance matrices can be generated by a finite number of the auto-correlations $R_{k}$. Hence, it is sufficient to work with a partial correlation parametrization of the spectrum $\Phi_{\chi_{o}}$.

From (28) it follows that

$$
R_{k}=\frac{1}{2 \pi} \int_{-\pi}^{\pi} \frac{\widetilde{\Phi}_{\chi_{o}}}{\left|\mathcal{F}_{D}\left(\mathrm{e}^{j \omega}\right)\right|^{2}} \mathrm{e}^{k j \omega} d \omega
$$

which together with

$$
\left|\mathcal{F}_{D}\left(\mathrm{e}^{j \omega}\right)\right|^{2}=\sum_{k=-m}^{m} f_{k} \mathrm{e}^{-k j \omega}
$$

gives

$$
\frac{1}{2 \pi} \int_{-\pi}^{\pi} \widetilde{\Phi}_{\chi_{o}} d \omega=\sum_{k=-m}^{m} f_{k} R_{k}
$$

The output energy (23) is now easily parametrized using (26) and (32) as

$$
\begin{aligned}
& \frac{1}{2 \pi} \int_{-\pi}^{\pi}\left|W_{y}\left(\mathrm{e}^{j \omega}\right)\right|^{2} \Phi_{y}(\omega) d \omega \\
= & \sum_{k=-m}^{m} f_{k}\left[\begin{array}{ll}
1 & 1
\end{array}\right] R_{k}\left[\begin{array}{l}
1 \\
1
\end{array}\right]
\end{aligned}
$$

Notice that (33) is a linear function in $R_{k}$.

We have presented a general finite linear parametrization of $P^{-1}$, see (29), that parametrizes all achievable covariance matrices. Here, we have considered a weighted output variance constraint. However, this type of parametrization can in principle handle all variance constraints that are linear in $\Phi_{\chi_{o}}$.

\subsection{A parametrization based on a finite spectrum}

So far we have considered parametrizations of $P^{-1}$ based on partial correlation parametrizations of the spectrum. Here we will introduce a useful and flexible parametrization based on a finite dimensional spectrum parametrization instead. Throughout this section 
we will assume that $G$ is stable and minimum phase. The input spectrum will be parametrized as

$$
\Phi_{u}(\omega)=\sum_{k=-M_{u}}^{M_{u}} r_{k} \mathcal{B}_{k}^{u}\left(\mathrm{e}^{j \omega}\right)
$$

for some stable basis functions $\mathcal{B}_{k}^{u}$ that we assume are such that $\mathcal{B}_{-k}^{u}=\left(\mathcal{B}_{k}^{u}\right)^{*}$. The cross spectrum is defined by

$$
\Phi_{u e}(\omega)=-\frac{H\left(\theta_{o}\right) \lambda_{o}}{G\left(\theta_{o}\right)} T\left(\theta_{o}\right)
$$

where $T$ is the complementary sensitivity function defined by $T=G K /(1+G K)$. Now introduce the parametrization

$$
\Phi_{u e}(\omega)=-\frac{H\left(\theta_{o}\right) \lambda_{o}}{G\left(\theta_{o}\right)} \sum_{k=0}^{M_{c}} s_{k} \mathcal{B}_{k}^{c}\left(\mathrm{e}^{j \omega}\right)
$$

where $\left\{\mathcal{B}_{k}^{c}\left(\mathrm{e}^{j \omega}\right)\right\}$ represents a set of stable basis functions. Notice that the parametrization in (36) corresponds to a linear and finite parametrization of $T$. For a sequence $\left\{s_{k}\right\}$, the controller $K$ is given by

$$
K(q)=\frac{\sum_{k=0}^{M_{c}} s_{k} \mathcal{B}_{k}^{c}\left(\mathrm{e}^{j \omega}\right)}{G\left(\theta_{o}\right)\left(1-\sum_{k=0}^{M_{c}} s_{k} \mathcal{B}_{k}^{c}\left(\mathrm{e}^{j \omega}\right)\right)}
$$

Remark: The controller $K$ in (37) provides an internally stable closed loop system. Furthermore, integral action in the controller can be imposed by the constraint $\sum_{k=0}^{M_{c}} s_{k} \mathcal{B}_{k}^{c}(1)=1$.

When the input spectrum and the cross spectrum are defined by (34) and (36), respectively, the inverse covariance matrix (14) is given by

$$
\begin{aligned}
P^{-1}\left(\theta_{o}\right)= & R_{o}\left(\theta_{o}\right)+\sum_{k=-M_{u}}^{M_{u}} r_{k} B_{P}^{u}(k) \\
& -\sum_{k=0}^{M_{c}} s_{k}\left(B_{P}^{c}(k)+\left(B_{P}^{c}(k)\right)^{T}\right)
\end{aligned}
$$

Notice that (38) is a linear and finite parametrization in $r_{k}$ and $s_{k}$. In (38) we have

$$
\begin{aligned}
& R_{o}\left(\theta_{o}\right)=\frac{1}{2 \pi} \int_{-\pi}^{\pi} \mathcal{F}_{e}\left(\mathrm{e}^{j \omega}, \theta_{o}\right) \mathcal{F}_{e}^{*}\left(\mathrm{e}^{j \omega}, \theta_{o}\right) d \omega \\
& B_{P}^{u}(k)= \\
& \frac{1}{2 \pi \lambda_{o}} \int_{-\pi}^{\pi} \mathcal{F}_{u}\left(\mathrm{e}^{j \omega}, \theta_{o}\right) \mathcal{B}_{k}^{u}\left(\mathrm{e}^{j \omega}\right) \mathcal{F}_{u}^{*}\left(\mathrm{e}^{j \omega}, \theta_{o}\right) d \omega
\end{aligned}
$$

and

$$
\begin{aligned}
& B_{P}^{c}(k)= \\
& \frac{1}{2 \pi} \int_{-\pi}^{\pi} \mathcal{F}_{u}\left(\mathrm{e}^{j \omega}, \theta_{o}\right) \mathcal{F}_{e}^{*}\left(\mathrm{e}^{j \omega}, \theta_{o}\right) \frac{H\left(\mathrm{e}^{j \omega}, \theta_{o}\right)}{G\left(\mathrm{e}^{j \omega}, \theta_{o}\right)} \mathcal{B}_{k}^{c}\left(\mathrm{e}^{j \omega}\right) d \omega
\end{aligned}
$$

The variance of $z_{y}=W_{y} y$, where $W_{y}$ is a stable linear filter and $y$ has the spectrum (24), can be expressed by the linear relation

$$
\begin{aligned}
& \frac{1}{2 \pi} \int_{-\pi}^{\pi}\left|W_{y}\left(e^{j \omega}\right)\right|^{2} \Phi_{y}(\omega) d \omega=\sum_{k=-M_{u}}^{M_{u}} r_{k} B_{y}^{u}(k) \\
& -\sum_{k=0}^{M_{c}} s_{k} B_{y}^{c}(k)+R_{v}\left(\theta_{o}\right)
\end{aligned}
$$

where

$$
\begin{aligned}
& B_{y}^{u}(k)=\frac{1}{2 \pi} \int_{-\pi}^{\pi}\left|W_{y}\left(e^{j \omega}\right) G\left(\mathrm{e}^{j \omega}, \theta_{o}\right)\right|^{2} \mathcal{B}_{k}\left(\mathrm{e}^{j \omega}\right) d \omega \\
& B_{y}^{c}(k)= \\
& \frac{1}{2 \pi} \int_{-\pi}^{\pi} \mid W_{y}\left(e^{j \omega}\right) \Phi_{v}(\omega)\left[\mathcal{B}_{k}^{c}\left(\mathrm{e}^{j \omega}\right)+\left(\mathcal{B}_{k}^{c}\right)^{*}\left(\mathrm{e}^{j \omega}\right)\right] d \omega
\end{aligned}
$$

and

$$
R_{v}\left(\theta_{o}\right)=\frac{1}{2 \pi} \int_{-\pi}^{\pi}\left|W_{y}\left(e^{j \omega}\right)\right|^{2} \Phi_{v}(\omega) d \omega
$$

where $\Phi_{v}=\left|H\left(\theta_{o}\right)\right|^{2} \lambda_{o}$.

In (38), we have presented another finite linear parametrization of $P^{-1}$, based on a finite spectrum parametrization of $\Phi_{\chi_{o}}$ represented by (34) and (36). Due to the restriction on the spectrum, this parametrization does in general not parametrize all covariance matrices. There is a trade-off between flexibility and complexity in the choice of $M_{u}$ and $M_{c}$. However, this class of parametrizations can handle a larger class of constraints. Beside variance constraints as (39) also point-wise constraints, see (18), can be treated, which is not the case for parametrizations based on partial correlations. Furthermore, as illustrated, it possible to e.g. impose certain characteristics of the closed loop and the controller directly in the design stage, see (36) and (37).

\subsection{Numerical Illustration}

Here we will use the parametrizations in Section 7.2 on a very simple example. Consider the autoregressive model

$$
\left(1-1.4 q^{-1}+0.45 q^{-2}\right) y(t)=u(t-1)+e(t)
$$

and consider the experiment design problem

$$
\min _{\Phi_{\chi_{o}}} \operatorname{det} P \quad \text { s.t. E } y^{2}(t) \leq 2
$$

which is non-convex. To reformulate this problem we will use a spectrum parametrization based on (34) and (36) with $\mathcal{B}_{k}^{u}=\mathcal{B}_{k}^{c}=\mathrm{e}^{-j k \omega}$. This leads to a linear parametrization of both $P^{-1}$, see (38), and the output variance, see (39). The final step to obtain a tractable optimization problem is to change the objective function to $-\log \operatorname{det} P$ that is convex in $P^{-1}$. This altogether gives the convex optimization problem

$$
\begin{aligned}
& \min _{r_{0}, \ldots, r_{n}, s_{0}, \ldots, s_{m}}-\log \operatorname{det} P \\
& \text { s.t. } \sum_{k=-n}^{n} r_{k} B_{y}^{u}(k)-\sum_{k=0}^{m} s_{k} B_{y}^{c}(k)+R_{v}\left(\theta_{o}\right) \leq \alpha
\end{aligned}
$$

in the new variables $\left\{r_{0}, \ldots, r_{n}, s_{0}, \ldots, s_{m}\right\}$. With $n=2$ and $m=1$ we obtain the solution $r_{0}=5.325$, $r_{1}=-2.66, r_{2}=0.45, s_{0}=1.4$ and $s_{1}=-0.45$ which gives $\operatorname{det} P=2 \cdot 10^{-9}$. This solution corresponds to a feedback loop with a minimum variance control law and a white noise reference signal. This is in complete line with a result in $(\mathrm{Ng}$ et al., 1977a) 
that states that a D-optimal design for AR-systems of the form (40) with constrained output variance can indeed be achieved under exactly these conditions. But this is not the only solution. Another solution can be represented by a controller with integral action. To illustrate this we use the possibility to impose integral action in the controller directly in the design stage. This can be achieved by the constraint

$$
\sum_{k=0}^{m} s_{k} \mathcal{B}_{k}^{c}(1)=1 .
$$

The optimization problem (41) together with the structural constraint (43) now yields the solution $r_{0}=$ 5.325, $r_{1}=-2.16, r_{2}=0, s_{0}=1.9$ and $s_{1}=-0.9$, which corresponds to an experiment design with $K=$ $\left(1.9-1.85 q^{-1}+0.45 q^{-2}\right) /\left(1-q^{-1}\right)$ and the optimal reference is integrated white noise. Furthermore the value of the objective function in the optimum is $\operatorname{det} P=2 \cdot 10^{-9}$, i.e. the same as for the solution with the minimum variance controller.

\section{SUMMARY}

We have extended results on optimal input design in open loop recently presented in (Jansson and Hjalmarsson, 2004b; Jansson and Hjalmarsson, 2004a) to the closed loop setting. When the controller is fix, it has been shown that it is straightforward to modify existing open loop design techniques to handle design of the reference spectrum.

For the case where the feedback mechanism is manipulable, i.e. both the input spectrum $\Phi_{u}$ and the cross spectrum $\Phi_{u e}$ are used as design variables, some modifications of the parametrizations of the inverse covariance matrix and signal constraints have to be performed. Here we have shown how to parametrize these quantities to fit into the previously derived framework.

\section{REFERENCES}

Bombois, X., G. Scorletti, M. Gevers, R. Hildebrand and P. Van den Hof (2004a). Cheapest open-loop identification for control. In: Proc. 43th IEEE Conf. on Decision and Control. Bahamas.

Bombois, X., G. Scorletti, M. Gevers, R. Hildebrand and P. Van den Hof (2004b). Least costly identification experiment for control. Automatica. Submitted.

Byrnes, C.I., S.V. Gusev and A. Lindquist (2001). From finite covariance windows to modeling filters: A convex optimization approach. SIAM Review 43, 645-675.

Forssell, U. and L. Ljung (2000). Some results on optimal experiment design. Automatica 36(5), 749756.

Gevers, M. and L. Ljung (1986). Optimal experiment designs with respect to the intended model application. Automatica 22, 543-554.

Goodwin, G.C. and R.L. Payne (1977). Dynamic System Identification: Experiment Design and Data Analysis, volume 136 of Mathematics in Science and Engineering. Academic Press.
Grenander, U. and G. Szegö (1958). Toeplitz Forms and Their Applications. University of California Press. Berkley, CA.

Gustavsson, I., L. Ljung and T. Söderström (1977). Identification of processes in closed loop identifiability and accuracy aspects. Automatica 13, 59-75.

Hildebrand, R. and M. Gevers (2003). Identification for control: Optimal input design with respect to a worst case $\nu$-gap cost function. SIAM Journal on Control and Optimization 41(5), 1586-1608.

Hjalmarsson, H., M. Gevers and F. De Bruyne (1996). For model-based control design, closed loop identification gives better performance. Automatica 32(12), 1659-1673.

Jansson, H. and H. Hjalmarsson (2004a). A general framework for mixed $\mathcal{H}_{\infty}$ and $\mathcal{H}_{2}$ input design. IEEE Trans. Aut. Contr. submitted.

Jansson, H. and H. Hjalmarsson (2004b). Mixed $\mathcal{H}_{\infty}$ and $\mathcal{H}_{2}$ input design for identification. In: CDC'04. Bahamas.

Lee, W. S., B. D. O. Anderson, R. L. Kosut and I. M. Y. Mareels (1993). A new approach to adaptive robust control. IJACSP 7, 183-211.

Lindqvist, K. and H. Hjalmarsson (2000). Optimal input design using linear matrix inequalities.. In: Proc. 12th IFAC Symposium on System Identification. Santa Barbara, California, USA.

Lindqvist, K. and H. Hjalmarsson (2001). Identification for control: Adaptive input design using convex optimization. In: Conference on Decision and Control. IEEE. Orlando, Florida, USA.

Ljung, L. (1985). Asymptotic variance expressions for identified black-box transfer function models. IEEE Transactions on Automatic Control AC30, 834-844.

Ljung, L. (1999). System Identification - Theory For the User, 2nd ed. PTR Prentice Hall. Upper Saddle River, N.J.

Ng, T. S., G. C. Goodwin and R. L. Payne (1977a). On maximal accuracy estimation with output power constraints. IEEE Trans. Automatic Control 22, 133-134.

Ng, T. S., G. C. Goodwin and T. Söderström (1977b). Optimal experiment design for linear systems with input-output constraints. Automatica 13, 571-577.

Ninness, B. and H. Hjalmarsson (2002). Exact quantification of variance error. In: 15th World Congress on Automatic Control. Barcelona, Spain.

Payne, R.L. and G.C. Goodwin (1974). Simplification of frequency domain experiment design for SISO systems. Technical Report 74/3. Imperial College, London.

Stoica, P. and T. Söderström (1982). A Useful Parameterization for Optimal Experiment Design. IEEE Trans. Aut. Contr. AC-27(4), 986-989.

Yuan, Z. D. and L. Ljung (1985). Unprejudiced optimal open loop input design for identification of transfer functions. Automatica 21(6), 697-708.

Zang, Z., R. R. Bitmead and M. Gevers (1995). Iterative weighted least-squares identification and weighted LQG control design. Automatica 31, 1577-1594.

Zarrop, M. (1979). Design for Dynamic System Identification. Lecture Notes in Control and Information Sciences. Sci. 21. Springer Verlag, Berlin. 